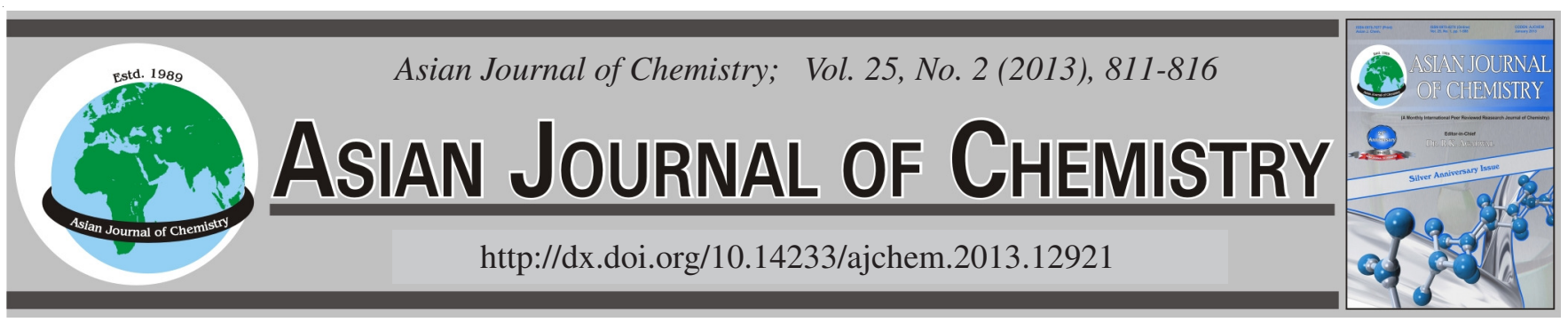

\title{
Protein Immobilization on A Biotinylated Polymer Coating
}

\author{
Jingzhe Zhang ${ }^{1}$, Xintong Wang ${ }^{2}$, Limei Liu ${ }^{3}$, Peimin Chen $^{3}$, Xiaohong Zhang $^{4, *}$ and Tiancheng Lu ${ }^{5}$
}

\author{
${ }^{1}$ Department of Orthopedics, China-Japan Union Hospital, Jilin University, Changchun 130033, P.R. China \\ ${ }^{2}$ Bethune Medical School, Jilin University, Changchun 130021, P.R. China \\ ${ }^{3}$ Jilin Province Power Hospital, Changchun 130022, P.R. China \\ ${ }^{4}$ School of Pharmaceutical Sciences, Jilin University, Changchun 130021, P.R. China \\ ${ }^{5}$ College of Life Sciences, Jilin Agricultural University, Changchun 130118, P.R. China \\ *Corresponding author: E-mail: xiaohongzhang88@ sina.com
}

Key Words: Poly(L-lactide), Biotin, Protein immobilization, Biocompatibility.

\section{INTRODUCTION}

Chemically designed surface coatings, which allow specific protein binding, are important in a variety of fields including immunoassay, protein separation and identification, cell assays, toxicological analysis, drug screening, biochip and clinic diagnostics ${ }^{1,2}$. Protein immobilization is often through the physical or chemical adsorption, physical embedding and the chemical interaction between surface and protein ${ }^{3-5}$. Many works have been devoted to preparing these surfaces, such as self-assembled monolayer on gold, glass, silicon, titanium and titanium oxide, polyelectrolyte multilayer coatings and hydrogels ${ }^{6,7}$. Usually these surfaces can not specifically bind proteins or interact in a biomimetic way with living cells. To do so, they should be modified with biomolecules to be capable of recognizing and combining with specific proteins or cells ${ }^{8}$. The well-designed immobilized technology is required in order that the surfaces allow proteins or bioolecules binding on the surface, avoid protein denaturation and inactivation and prevent the interference with target protein. Several groups have attached biological moieties onto synthetic nondegradable polymers ${ }^{3-5}$. However, degradability may be important so that implanted cells and proteins can eventually obtain a completely natural environment, thereby eliminating the possibility of long-termed detrimental tissue response ${ }^{9}$. Huang et al. ${ }^{1}$, synthesized biotinylated poly (L-lysine)-g-poly(ethylene glycol) and attached it onto negative-charged oxide surfaces to form bioaffinity sensor. However, the method needs negatively charged substrate and the electrostatic forces between the polymer and the substrate are not strong and may be affected by the change of $\mathrm{pH}$, ionic strength and charged bio-molecules. Therefore, preparation of a bioactive surface can recognize and combine with specific proteins or cells and at the same time repel unwanted proteins or cells is a severe challenge for both bio-scientists and material scientists.

In the present study, we attempted to construct such a surface by coating a layer of biotinylated poly(ethylene glycol)-b-poly(L-lactide)-b-poly(L-lysine) (PEG-PLA-PLL/ biotin) on a substrate and covering it with a layer of blocking agent consisting of gelatin, poly(N-vinyl pyrrolidone) and casein. Its bioactivity comes from the biotin-streptavidin interaction. Because the activity unit (double five-member heterocyclic rings) of biotin and the activity cavity of streptavidin are similarly a "key and lock" relationship in the spatial structure and form the hydrogen bonding ${ }^{10}$. The interaction between biotin and streptavidin is one of the strongest noncovalent 
forces in nature $\left(\mathrm{K}_{\mathrm{aff}}=10^{15} / \mathrm{M}^{-1} \text { in solution }\right)^{11}$ and thus the biotin-streptavidin complexation can be regarded to be complete and irreversible. A streptavidin has four subunits, carrying four same active sites for biotin binding. As an active site bind to biotin, the rest sites can still interact with other biotin. With the PEG-PLA-PLL/biotin layer, therefore, the surface can specifically combine with streptavidin and as a result of this combination, the surface can further combine with any biotinylated bio-moieties via the biotin-streptavidin bridges. This polymer coating combines biodegradability, biocompatibility, amphiphilic properties and bio-activity. In the present paper, the preparation and evaluation of such polymer coating is presented.

\section{EXPERIMENTAL}

Dicyclohexylcarbodiimide (DCC) from GL Biochem (Shanghai) Ltd. was used as received. N-Hydroxysuccinimide (NHS) was purchased from Fluka, USA. Dimethyl sulfoxide was refluxed over $\mathrm{CaH}_{2}$ and distilled under nitrogen. DMF was dried over $\mathrm{CaH}_{2}$ and distilled before use. The diblock copolymer methoxy-poly(ethylene glycol)-b-poly(L-lactide) (PEG-PLA, DP = 17, 23, i.e., degrees of polymerization of PEG block was 17 and that of PLA block was 23) and triblock copolymer methoxy-poly(ethylene glycol)-b-poly (L-lactide)b-poly(L-lysine) (PEG-PLA-PLL, DP = 17, 23, 14) was synthesized in our lab as described previously ${ }^{12}$. Biotinylated rabbit antigoat monoclonal antibodies (biotin/Ab) were obtained from Vector Lab. Inc. (Burlinggame, CA, USA); streptavidin and fluorescein isothiocyanate (FITC) labeled goat globulin (GLB/FITC) were supplied by Promega (Madison, WI, USA); gelatin was purchased from Beijing Huateng Chemical, China; milk casein, poly(N-vinyl pyrrolidone) (PVP) with a molecular weight of $360 \mathrm{kDa}$ and poly(L-lysine) with a molecular weight of $150-300 \mathrm{kDa}$ were supplied by Sigma. These reagents were used without further purification.

Syntheses of biotinylated PEG-PLA-PLL (PEG-PLAPLL/biotin): As in Scheme-I, the PEG-PLA-PLL was biotinylated by first activating biotin with NHS and then coupling with the $\mathrm{NH}_{2}$ groups on PEG-PLA-PLL. According to reference $^{13}$, the biotin-N-hydroxy succinimide was prepared from biotin and N-hydroxy succinimide with the help of dicyclohexylcarbodiimide. $1 \mathrm{~g}$ of biotin $(4 \mathrm{mmol})$ was dissolved in $20 \mathrm{~mL}$ of hot DMF and cooled to room temperature and 6 mmol of N-hydroxy succinimide was added under stirring. In a separate flask, $0.8 \mathrm{~g}$ of dicyclohexylcarbodiimide ( $4 \mathrm{mmol})$ was dissolved in $2 \mathrm{~mL}$ of $\mathrm{DMF}$ and the solution was added to the biotin/ $\mathrm{N}$-hydroxy succinimide mixture. The reaction mixture was stirred overnight at room temperature and the precipitate dicyclohexylurea (DCU) was filtered out. The filtrate was precipitated with an excess of ether. The product (biotin-N-hydroxy succinimide) was further purified with 2-propanol, dried under vacuum at $40{ }^{\circ} \mathrm{C}$ for $24 \mathrm{~h}$ and then kept in a refrigerator.

For coupling of biotin, $100 \mathrm{mg}$ of PEG-PLA-PLL (0.015 $\mathrm{mmol})$ and $140 \mathrm{mg}$ of biotin-NHS $(0.40 \mathrm{mmol})$ were dissolved in $2 \mathrm{~mL}$ anhydrous DMF. After adding $0.07 \mathrm{~mL}$ of triethylamine, the reaction mixture was stirred at room temperature for $24 \mathrm{~h}$. The product mixture was precipitated in excessive ether and then dialyzed against water with a cellulose
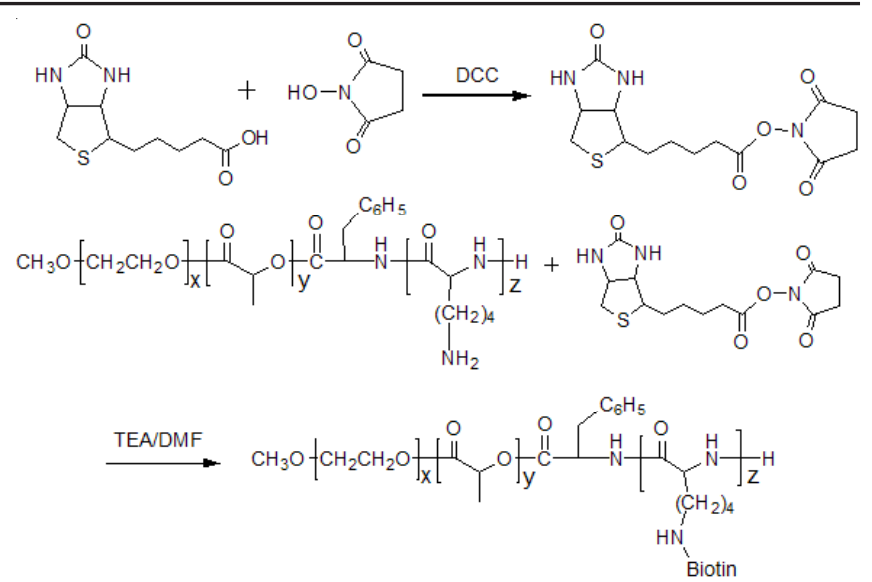

Scheme-I: Synthesis of copolymer PEG-PLA-PLL/biotin

membrane (cut-off Mn 3500) for 2 days. After dialysis, the solution was immediately lyophilized for 2 days. FT-IR spectra were recorded on a Bio-Rad Win-IR instrument. ${ }^{1} \mathrm{H}$ NMR spectra were measured by an AV-400 NMR spectrometer at room temperature.

A known amount of dried PLGA-PEG-PLL/biotin was dissolved in dimethyl sulfoxide. The UV absorbance at $250 \mathrm{~nm}$ was measured by a UV-VIS spectrophotometer (UV-2401PC, Shimadzu) to determine the content of conjugated biotin. Serially diluted concentrations of biotin in DMSO were used to construct a calibration curve of biotin.

Formation of polymer coatings: Glass slides were used as the substrate for PEG-PLA-PLL/biotin coating. The glass slides were rinsed sequentially with basic lotion, acid lotion and distilled water and degreased with $95 \%$ alcohol. After drying, the glass slides were enveloped with $500 \mu \mathrm{L}$ of sigma poly(L-lysine) solution at a concentration of $0.1 \mathrm{~g} / \mathrm{L}$ so as to bond the polymer onto the substrate tightly and avoid polymer peeling ${ }^{14}$. The PEG-PLA-PLL/biotin was dissolved in DMF completely with a concentration of $0.1 \mathrm{~g} / \mathrm{L}$ and $500 \mu \mathrm{L}$ of the solution was cast on the substrate. Control coatings were prepared in the similar way from copolymers PEG-PLA and PEG-PLA-PLL. The coated slides were kept under vacuum for $48 \mathrm{~h}$ or more to remove the last traces of DMF.

Surface blocking of the coatings: A blocking agent was prepared by dissolving $500 \mathrm{mg}$ of gelatin, $20 \mathrm{mg}$ of PVP and $5 \mathrm{mg}$ of casein in $100 \mathrm{~mL}$ of $0.05 \mathrm{~mol} / \mathrm{L}$ Tris- $\mathrm{HCl}$ solution in a water bath of $30^{\circ} \mathrm{C}$ to obtain a final $\mathrm{pH}$ value of 8.4 as used in reference ${ }^{15}$. In experiments, $500 \mu \mathrm{L}$ of the blocking agent was cast on PEG-PLA, PEG-PLA-PLL and PEG-PLA-PLL/ biotin coatings via $2 \mathrm{~h}$ incubation at room temperature. These blocked coatings were dried under vacuum finally.

Non-specific adsorption and its blocking: Meanwhile, blocked polymer coatings were incubated in $1 \mathrm{~mL}$ of 0.02 mol/L PBS containing $0.2 \mu \mathrm{g}$ of GLB/FITC, with non-blocked coatings as control. And then they were thrice rinsed thoroughly with PBST, 5 min for each. Finally, the samples were washed thoroughly with triply distilled water, dried in air and observed under a confocal laser scanning microscope (CLSM, Leica TCS SP2).

Charaterization of polymer coatings: The above nonblocked or blocked polymer coatings were characterized as follows. Their surface energy spectra were determined by an 
X-ray photoelectron spectroscopy (XPS, Escalab250, Thermo). Their thickness was measured by Stylus Profiler (Dektak 6M, Veeco). Their wettability was investigated by determining water contact angle with a Drop Shape Analyser (DSA 10, Krüss GmbH). Morphologies of PEG-PLA-PLL/ biotin coatings were imaged by both Atomic Force Microscopy (SPI 3800/SPA 300 HV, Seiko Instruments, Japan) and Environmental Scanning Electron Microscopy (Model XL 30 ESEM FEG, Micro FEI Philips).

Fluorescence associated immunoassay (FIA): FIA was a method to detect the specific combination of biotinylated copolymer with streptavidin, streptavidin with biotin/Ab and biotin/Ab with GLB/FITC) as shown in Fig. 1. Briefly, the biotinylated copolymer coating was blocked and incubated with $0.3 \mu \mathrm{g}$ of streptavidin. After the streptavidin-combined coatings were blocked, subsequential incubations in $0.2 \mu \mathrm{g}$ of biotin/Ab and $0.2 \mu \mathrm{g}$ of GLB/FITC were performed. Each bio-sample was dissolved in $1 \mathrm{~mL}$ of $0.02 \mathrm{~mol} / \mathrm{L}$ PBS and each incubation was followed by thrice rinses with PBST, $5 \mathrm{~min}$ for each. Finally, the samples were analyzed by a CLSM after thorough rinse with triply distilled water.

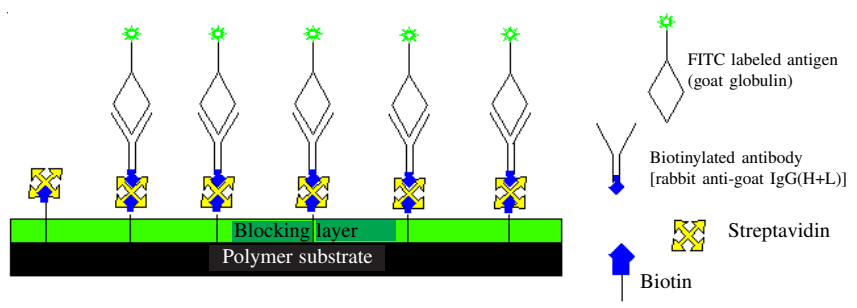

Fig. 1. Schematic illustration of the bioactive surface and its confocal laser scanning microscope (CLSM) analysis

Cell adhesion and spreading: L929 fibroblasts were chosen as test cells. The cells were purchased from Shanghai Institute of Cell Biology, Chinese Academy of Sciences and cultured with Dulbecco's modified Eagle's medium (DMEM, GIBCO) supplemented with $10 \%$ fetal bovine serum (FBS, GIBCO), $1.0 \times 10^{5} \mathrm{U} / \mathrm{L}$ penicillin (Sigma) and $100 \mathrm{mg} / \mathrm{L}$ streptomycin (Sigma). The copolymer PEG-PLA-PLL/biotin was dissolved in DMF and cast on cover slides. Pure PLA coating was prepared in a similar way and was used as control. The coated slides were kept under vacuum for $48 \mathrm{~h}$ to remove the last trace of DMF and exposed to UV light for $0.5 \mathrm{~h}$ for sterilization. After they were placed in each well of 6-well tissue culture plates (NUNC), the L929 cells were seeded on the cover slides at a density of $2 \times 10^{5}$ cells/well, incubated in a humidified incubator at $37{ }^{\circ} \mathrm{C}$ and $5 \% \mathrm{CO}_{2}$. The picture of each cover slide was taken with a digital camera (DXM1200F, Nikon) after 8 h, $20 \mathrm{~h}$ and $2 \mathrm{D}$, respectively.

\section{RESULTS AND DISCUSSION}

Synthesis of PEG-PLA-PLL/biotin: Prior to conjugate biotin to triblock copolymer PEG-PLA-PLL, biotin was firstly activated with $\mathrm{N}$-hydroxysuccinimide in the presence of dicyclohexylcarbodiimide, as shown in Scheme-I. The structure of the biotin-NHS ester was confirmed by ${ }^{1} \mathrm{H}$ NMR as shown in Fig. 2a, i.e., the signals of the two methine protons ( $\mathbf{m}$ at $4.2 \mathrm{ppm}, \mathbf{n}$ at $4.3 \mathrm{ppm}$ ) and the two urea protons ( $\mathbf{p}$ at
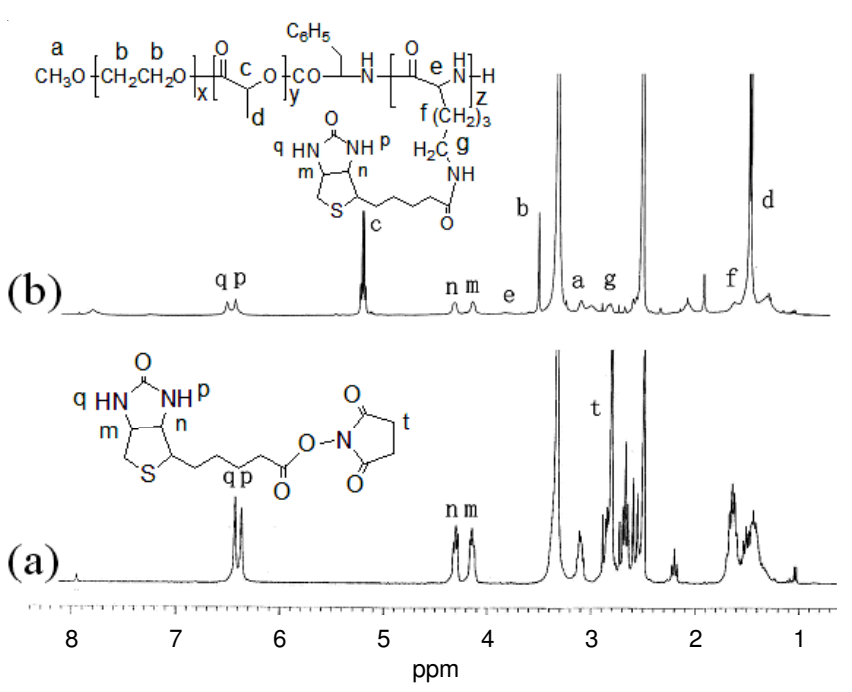

Fig. 2. $\quad{ }^{1}$ H NMR spectra and their assignments of (a) Biotin-NHS, (b) PEGPLA-PLL/Biotin

$6.35 \mathrm{ppm}, \mathbf{q}$ at $6.45 \mathrm{ppm}$ ) of the cyclic biotin structure as well as the methylene peak at $2.95 \mathrm{ppm}$ of the succinimide ring.

PEG-PLA-PLL/biotin was prepared by reacting biotinNHS ester with copolymer PEG-PLA-PLL as shown in Scheme-I. The ${ }^{1} \mathrm{H}$ NMR spectrum (Fig. 2b) of the product showed the characteristic proton peaks of biotin, such as the two methine protons ( $\mathbf{m}$ at $4.2 \mathrm{ppm}, \mathbf{n}$ at $4.3 \mathrm{ppm}$ ) and the two urea protons (p at $6.35 \mathrm{ppm}, \mathbf{q}$ at $6.45 \mathrm{ppm}$ ), but the characteristic proton peak of succinimide disappeared.

The biotin number grafted on the polymer could be calculated from the integral ratio of peak $\mathrm{m}$ ( or $\mathbf{n}, \mathbf{p}, \mathbf{q}$ ) to peak $\mathbf{b}\left(\mathrm{CH}_{2}\right.$ protons of the PEG block at $\left.3.51 \mathrm{ppm}\right)$. As a result, 90.1\% PLL units were biotinylated. The structure was further confirmed by FT-IR spectrum (Fig. 3). The coexistence of the peak at $1680 \mathrm{~cm}^{-1}$ (amide group of the biotin ring) and the peak at $1734 \mathrm{~cm}^{-1}$ (ester group of the PLA block) and the relative intensity change in the $\mathrm{NH}$ bands at $3435 \mathrm{~cm}^{-1}\left(\mathrm{v}_{\mathrm{NH}_{2}}\right.$ of the PLL block) and $3300 \mathrm{~cm}^{-1}$ ( $\mathrm{V}_{\mathrm{NH}}$ of the amide groups formed) from Fig. 4C-B indicated that the copolymer had been biotinylated successfully.

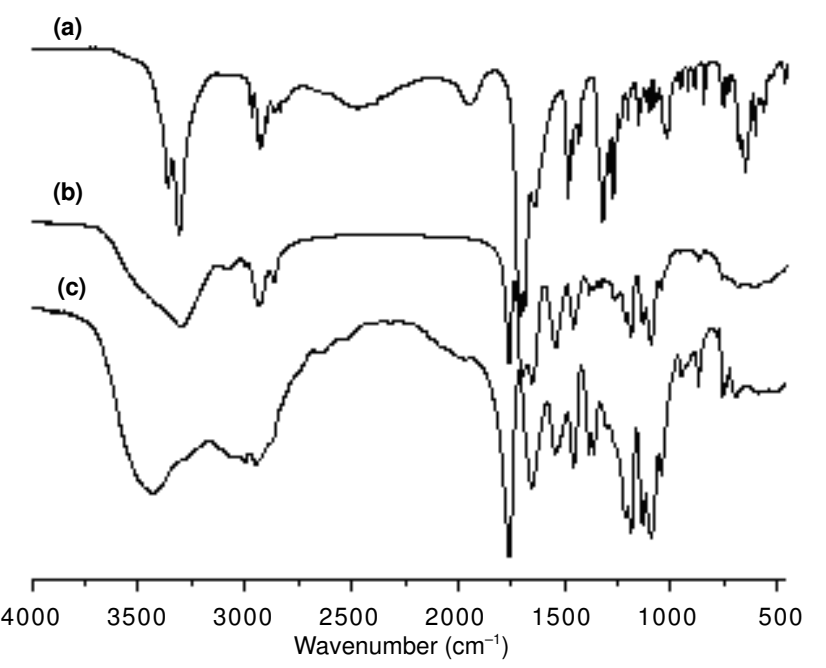

Fig. 3. IR spectra of (a) Biotin, (b) PEG-PLA-PLL/biotin, (c) PEG-PLAPLL 
$0.25 \mathrm{mg} / \mathrm{mL}$ of biotinylated copolymer has a mean UV absorbance value of 0.4389 at $250 \mathrm{~nm}$. Based on predetermined standard curve formula $\mathrm{y}=0.0116+4.1099 \mathrm{x}$ (x: biotin concentration in $\mathrm{mg} / \mathrm{mL}$; $\mathrm{y}$ : optical density), the graft ratio of biotin was $91.5 \%$. That is to say, 12.8 molecules of biotin were conjugated to the 14 lysine residues in one PEG-PLA-PLL molecule. The graft ratio was in agreement with the NMR result.

Non-specific adsorption and its blocking: GLB/FITC was chosen as a model protein to examine its nonspecific adsorption on polymer surfaces. The polymer coatings were incubated in GLB/FITC solution, followed with thorough rinses. Provided GLB/FITC is adsorbed on the surface, fluorescence would be confirmed by CLSM analysis on the surface. As shown in Fig. 4a, the green fluorescence was quite strong on PEG-PLA-PLL/biotin, PEG-PLA coating did not show appreciable fluorescence and PEG-PLA-PLL adsorbed more than the other two. Obviously, the strong fluorescence was a nonspecific adsorption because there were no specific interactions between the polymer surface and GLB/FITC.This undesirable nonspecific binding would bring about falsepositive results and thus severely interfere with the subsequent analysis. Therefore, it should be eliminated or minimized.

In the literatures, several blocking agents are used to remove undesirable nonspecific adsorption of proteins. Their function is to saturate the remaining adsorptive sites on polymer surface. Some inert proteins, non-ionic detergents and polymers can play this role ${ }^{16-18}$. The choice of blocking agents is usually based on empirical testing ${ }^{16}$ by considering (1) the nature of surface, (2) the type of bio-molecules to be immobilized to the surface, (3) the type of detective probe or system employed. Among the blocking agents reported, Tween 20, casein, gelatin and poly(N-vinyl pyrrolidone) (PVP) are typical examples ${ }^{16}$. Tween 20 is a non-ionic surfactant and can lower down the interaction between hydrophobic proteins and polymer surface. Casein and gelatin are inert proteins. They usually do not involve in immunological processes but they are effective in obstacling nonspecific protein-protein interaction (gelatin) and protein-solid phase adsorption (casein) ${ }^{17}$. PVP is a water soluble polymer and it can be coated easily onto hydrophobic surfaces and render them both non-binding and hydrophilic ${ }^{18}$. However, they have disadvantages if they are used individually. For example, their blocking efficiency is limited in case both protein-polymer and protein-protein interactions coexist. Therefore, these ingredients, $0.5 \%$ gelatin, $0.02 \% \mathrm{PVP}$ and $0.005 \%$ casein (gelatin/PVP/casein) were mixed together in $0.05 \mathrm{~mol} / \mathrm{L}$ Tris $-\mathrm{HCl}$ solution in the present study to serve as the blocking agent.

As shown in Fig. 4b, after blocking with gelatin/PVP/ casein solution, incubation in GLB/FITC solution, blocked PEG-PLA-PLL/biotin and blocked PEG-PLA-PLL showed weak fluorescence in Fig. 4b when compared with Fig. 4a, while blocked PEG-PLA coating did not show fluorescence at all (Fig. 4b). These results showed that GLB did not adsorb on the polymer coatings due to the blocking effect of the gelatin/ $\mathrm{PVP} /$ casein layer. Therefore, the gelatin/PVP/casein solution was effective in eliminating nonspecific binding of proteins.

Nonspecific binding might involve hydrogen bonding, ionic binding and/or hydrophobic interaction between protein

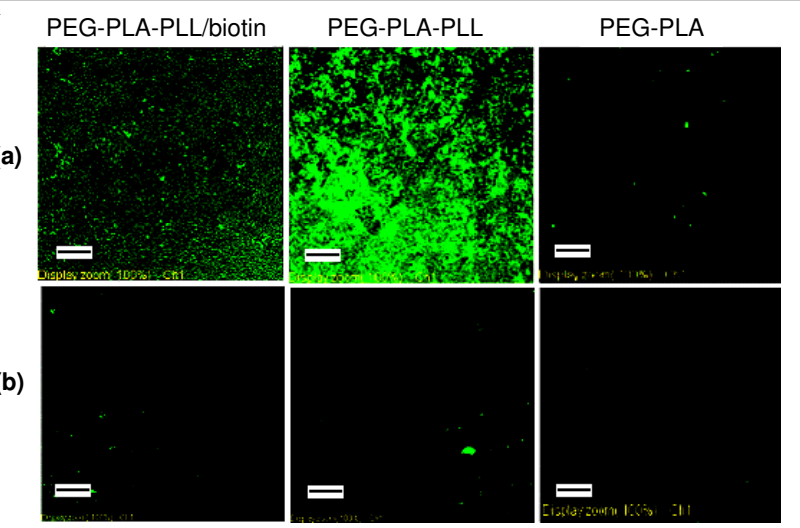

Fig. 4. Results of blocking effect in eliminating non-specific adsorption. Different polymer coatings incubated in GLB/FITC solution: (a) incubated before blocking; (b) incubated after blocking with gelatin/ $\mathrm{PVP} /$ casein solution. Bar is $1 \mu \mathrm{m}$

and polymer surface. On the experimental results (Fig. 4a), PEG chains are protein repellent ${ }^{19}$. This is the reason for low fluorescence on PEG-PLA. Both PEG-PLA-PLL and PEGPLA-PLL/biotin contain side-chain $\mathrm{NH}_{2}$ groups and are hydrophobic to some extent (Table-1) and thus there may be hydrogen bonding and hydrophobic interaction. The observed intense fluorescence of both PEG-PLA-PLL and PEG-PLAPLL/biotin coatings implies that affinity of both PLL and PLL/ biotin blocks with GLB/FITC overwhelms the repellence from the PEG block. Polymer PEG-PLA-PLL exhibits more nonspecific protein adsorption because it has more $\mathrm{NH}_{2}$ groups than PEG-PLA or PEG-PLA-PLL/biotin. The function of the blocking layer to get rid of the nonspecific binding is explained as follows. The components of our blocking agent, gelatin, PVP and casein, can participate in these interactions to saturate possible nonspecific adsorptive sites on the polymer surface. Therefore, when GLB/FITC was applied onto the surface, it could not replace the blocking molecules and eventually was rinsed out.

TABLE-1

COATING THICKNESSES AND CONTACT ANGLES

\begin{tabular}{lccc}
\hline \multicolumn{1}{c}{ Materials } & Blocking & $\begin{array}{c}\text { Coating thickness } \\
(\mathrm{nm})\end{array}$ & $\begin{array}{c}\text { Contact } \\
\text { angle }\left(^{\circ}\right)\end{array}$ \\
\hline Poly(L-lysine) & - & $3238 \pm 51$ & - \\
\hline \multirow{2}{*}{ PEG-PLA } & Before & $251 \pm 23$ & $57.2 \pm 1.1$ \\
& After & $444 \pm 16$ & 0 \\
\hline \multirow{2}{*}{ PEG-PLA-PLL } & Before & $252 \pm 11$ & 0 \\
& After & $462 \pm 21$ & 0 \\
\hline PEG-PLA- & Before & $286 \pm 19$ & $64.1 \pm 1.5$ \\
PLL/biotin & After & $507 \pm 20$ & 0 \\
\hline
\end{tabular}

Characterization of polymer coatings: Since the nonspecific binding was eliminated efficiently, there ought to be some changes on the coating surface after blocking. Thus some characterization of the coatings was carried out to reveal such changes.

The coating thickness was measured by a Stylus Profiler: Table-1 showed typical thickness data of the coatings prepared. The Sigma poly(L-lysine) undercoating was $3.23 \mu \mathrm{m}$. PEGPLA-PLL/biotin coating was $286 \mathrm{~nm}$ before blocking and 507 $\mathrm{nm}$ after blocking. The thickness of the blocking layer was 
approximately $221 \mathrm{~nm}$. Similarly, the blocking layers on PEGPLA and PEG-PLA-PLL were 193 and $210 \mathrm{~nm}$.

The surface energy spectrum of PEG-PLA and PEG-PLAPLL/biotin was measured by XPS to investigate the surface elemental composition before and after blocking. The surface contents of carbon, oxygen, nitrogen and sulfur for the nonblocked PEG-PLA-PLL/biotin were 71.2, 27.0, 1.2 and 0.6 , respectively. The presence of nitrogen and sulfur was attributed to the PLL/biotin block rather than the Sigma poly(Llysine) undercoating, because the PEG-PLA coating gave an elemental composition of 78.5, 21.5, 0 and 0 although it was thinner than PEG-PLA-PLL/biotin coating (251 nm versus 286 $\mathrm{nm}$, Table-1) and there was an identical Sigma poly(L-lysine) coating under it. In other words, the XPS technique can reveal the elemental composition in the outmost layer of the coating. After blocking, the element composition of PEG-PLA-PLL/ biotin became 70.1, 23.1, 6.2 and 0.6. The nitrogen content was increased while the oxygen content decreased relatively, due to the more nitrogen-to-oxygen ratio in the blocking layer when compared to the underneath layer.

The surface change after blocking was also supported by water contact angle measurement. As shown in Table-1, the contact angles on PEG-PLA and PEG-PLA-PLL/biotin coatings before blocking were 57.2 and $64.1^{\circ}$, respectively; while they were close to zero degree after blocking, due to the excellent water solubility of gelatin, PVP and casein in the blocking layer. The PEG-PLA-PLL coating did not display water wettability improvement after blocking, because its original water contact angle was very low due to the hydrophilic amino side groups in lysine residues. In addition, AFM and ESEM observations showed that the surface of PEG-PLA-PLL/ biotin coating after blocking was continuous, smooth and dense (image is omitted). It is important to declare that the defatted glass slides used in this experiment was not silicified ${ }^{20}$, but coated with the Sigma lysine polymer ${ }^{14}$. In the experimental process, there was not the polymer coatings' peeling or was not the nonspecific adsorption onto the Sigma lysine base (data is omitted).

Fluorescence associated immunoassay (FIA): The FIA was performed to examine specific protein binding. For this purpose, biotin/Ab and GLB/FITC were chosen as one pair of antibody and antigen and FITC as fluorescent marker. As in Fig. 5, after PEG-PLA-PLL/biotin coating was blocked and incubated in streptavidin, biotin/Ab and GLB/FITC solution consecutively, uniform strong fluorescence was observed on the surface, indicating that a series of molecular processes had taken place on the polymer surface. They were specific recognition and non-covalent binding of biotin-streptavidin, streptavidin-biotin/Ab and Ab-GLB. It could be concluded that the gelatin/PVP/casein blocking layer inhibited the nonspecific binding of proteins, but did not obstacle the specific interaction between biotin and streptavidin and the combined streptavidin retained its own biological activity.

It is interesting to notice that the existence of the blocking layer on the PEG-PLA-PLL/biotin coating did not obstacle the specific binding of biotin and streptavidin. It means that the biotin heads on the PLL chains could approach the active site of streptavidin and form complex with it. This is because the blocking agent is composed of water soluble inert agents,

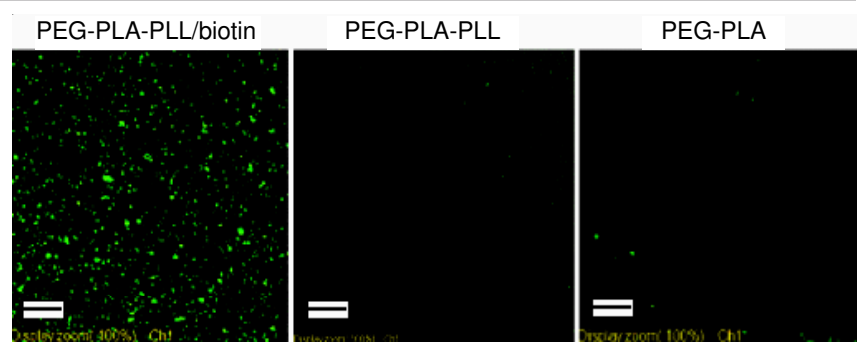

Fig. 5. Fluorescence associated immunoassay results of different polymer coatings blocked with gelatin/PVP/casein solution, incubated consecutively in streptavidin, biotin/Ab and GLB/FITC solutions. Bar is $1 \mu \mathrm{m}$

i.e., gelatin, PVP and casein and they can not interact with the bio-molecules involved in FIA but can help them renature ${ }^{16}$. Meanwhile, the blocking agent can be swollen by water under the reaction condition. Both biotin-carrying PLL chains and streptavidin molecules can diffuse into the blocking layer, recognize each other and form a complex there. Because the complexation constant of biotin and streptavidin is very high ${ }^{11}$, it is difficult to dissociate them once they are formed. In contrast, blocked PEG-PLA-PLL and PEG-PLA coatings did not show any fluorescence after the same treatments (Fig. 5) because of lacking biotin moieties.

Obviously, existence of biotin in the polymer coating is the necessary requirement for the specific combination. Without it, streptavidin can not anchor on the surface and consequently, biotin/Ab and GLB/FITC can not adhere to the surface. The strong biotin-streptavidin interaction is due to the shape-specificity of the biotin-binding pocket in streptavidin, which allows the formation of multiple hydrogen bonds and van der Waals interaction ${ }^{10}$. Since its tetrameric structure, streptavidin contains four equivalent biotin-binding sites, the streptavidin molecules immobilized on the surface retain the ability to bind other biotins. So it is convenient to immobilize other biotinylated molecules at the vacant sites of streptavidin. When compared to binding through covalent attachment of ligands to the bulk polymer, such binding is achieved without significant perturbation of the tertiary or quaternary structures of proteins and therefore it is stable over a wide range of $\mathrm{pH}$ and temperature ${ }^{10}$. Furthermore, it is universally applicable to any class of bio-molecules (e.g., proteins, peptides, oligosaccharides which can be biotinylated) and does not damage the anchored bio-molecules or the polymer substrate. Therefore, the above bioactive surface with biotin or with biotin/ streptavidin species may exhibit various biological functions, depending on what bio-molecules are attached to it.

Lu et al. ${ }^{19}$ have prepared similar polymer coatings. They used a solid phase enzyme linked immunosorbent assay (EIA) method to detect the specific protein immobilization. The enzymatic method depends on the toxic chromophore substrates, the colour development is time-dependent and the outcome decision depends on the discretionary subjectivity. In the present study, the detection method was improved that fluorescence was detected by CLSM in order that the outcome decision was directly objective. Even the fluorescence intensity can be quantified to fulfill the quantitatively high quality. Cell adhesion and spreading. Cell adhesion of various coatings was evaluated by culturing L929 cells in a culture medium of 
DMEM containing $10 \%$ of FBS. The test samples were PEGPLA-PLL and PEG-PLA-PLL/biotin copolymers and the control sample was pure PLA. Fig. 6 showed that the cell morphology during the incubation. After incubation for $12 \mathrm{~h}$, most of the cells on the copolymer PEG-PLA-PLL/biotin began to spread, while cells on PEG-PLA-PLL and PLA coatings were less and smaller. After incubating for $48 \mathrm{~h}$, the cells on the PEG-PLA-PLL/biotin coatings almost occupied the whole surface. The cells adhered and spread better and proliferated faster on PEG-PLA-PLL/biotin coatings than those on PEGPLA-PLL and PLA coatings, which may be ascribed to the presence of biotin as a nutritious material for cells and to the improved hydrophilicity caused by the PEG and PLL/biotin blocks. The result indicated that the copolymer PEG-PLAPLL/biotin had excellent biocompatibility and could be a promising biodegradable polymer matrix for biosensors ${ }^{21}$.

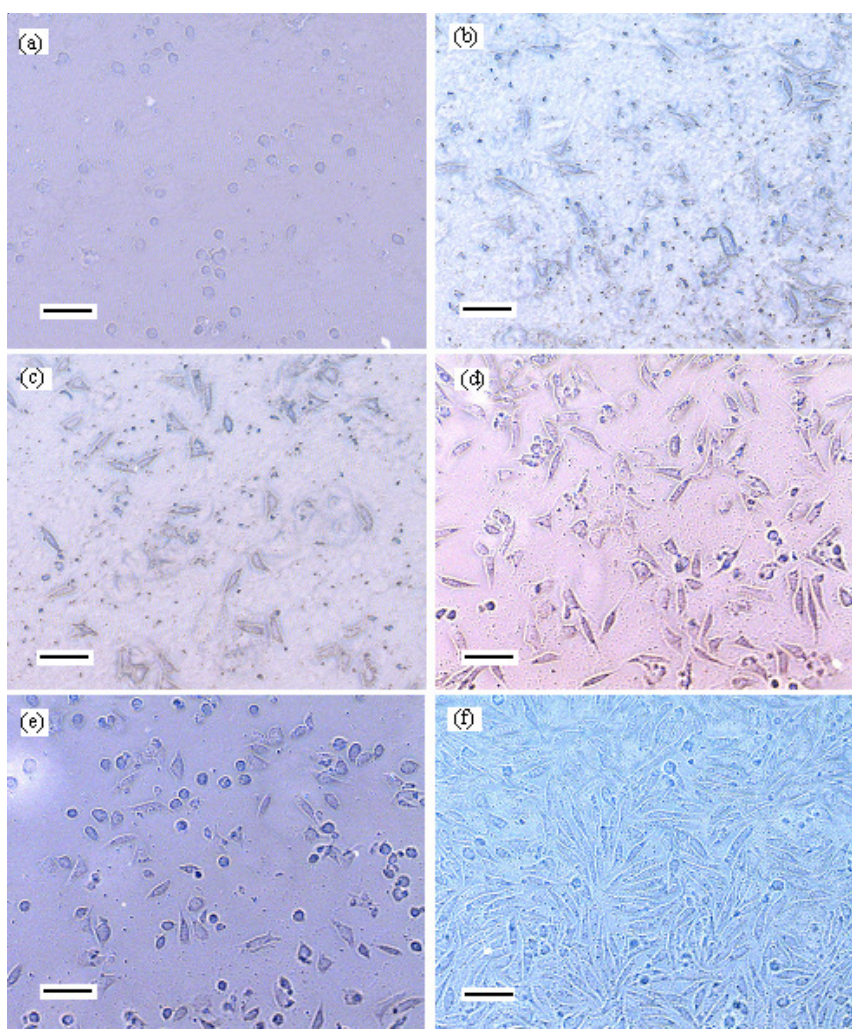

Fig. 6. Microscopic images of adhered and spread L929 cells. Coating samples: $a$ and b, PLA; $c$ and d, PEG-PLA-PLL; e and f, PEGPLA-PLL/biotin. Incubation time: (a, c and e), $12 \mathrm{~h}$; (b, d and f), $48 \mathrm{~h}$. Bar is $60 \mu \mathrm{m}$

\section{Conclusion}

A novel bioactive surface without nonspecific binding of proteins was prepared from a biodegradable amphiphilic triblock copolymer, biotinylated poly(ethylene glycol)-bpoly(L-lactide)-b-poly(L-lysine) (PEG-PLA-PLL/biotin) by coating it on a substrate and covering it with a layer of blocking agent consisting of gelatin, poly(N-vinyl pyrrolidone) and casein. The amount of biotin at the interface could be easily controlled by adjusting the chain length of PLL or the graft ratio of biotin. Nonspecific protein adsorption was excluded with PEG and gelatin/PVP/casein completely while specific bioaffinity was achieved by using streptavidin as a bridge between biotinylated polymer surfaces and biotinylated biomolecules. Based on the bio-molecules attached, the surface can exhibit corresponding bio-functions such as antigenantibody or ligand-receptor recognition and combination. Such bioactive surface also shows enhanced adhesion, spreading and proliferation of L929 cells. Therefore, it can be used in the fields of protein immobilization, biosensor, tissue engineering and target drug delivery.

\section{REFERENCES}

1. N.-P. Huang, J. Vörös, S.M. DePaul, M. Textor and N.D. Spencer, Langmuir, 18, 220 (2002)

2. J.G. Groll, E.V. Amirgoulova, T. Ameringer, C.D. Heyes, C. Rocker, G.U. Nienhaus and M. Moller, J. Am. Chem. Soc., 126, 4234 (2004).

3. G.W. Fussell and S.L. Cooper, Biomaterials, 25, 2971 (2004).

4. Z. Zhang, S.F. Chen and S.Y. Jiang, Biomacromolecules, 7, 3311 (2006).

5. S. Ko and J. Jang, Biomacromolecules, 8, 1400 (2008).

6. S. Tosatti, R. Michel, M. Texxtor and N.D. Spencer, Langmuir, 18, 3537 (2002).

7. K.Y. Lee and D.J. Mooney, Chem. Rev., 101, 1869 (2001).

8. K. Huang, B.P. Lee, D.R. Ingram and P.D. Messersmith, Biomacromolecules, 3, 397 (2002).

9. D. Anderheiden, O. Brenner, D. Klee, R. Kaufmann, H.A. Richter, P.T Bury and R. Langer, J. Am. Chem. Soc., 115, 11010 (1993).

10. C. Rosano, P. Arioso and M. Bolognes1, Biomol. Eng., 16, 5 (1999).

11. N.M. Green, Avidin, in Advances in Protein Chemistry, Academic Press, New York, Vol. 29, pp. 85-133 (1975).

12. C. Deng, X. Chen, H. Yu, J. Sun, T. Lu and X. Jing, Polymer, 48, 139 (2007).

13. M. Wilchek and E.A. Bayer, Methods Enzymol., 184, 123 (1990).

14. W.M. Huang, S.J. Gibson, P. Facer, J. Gu and J.M. Polak, Histochem., 77, 275 (1983).

15. D. Li, M.W. Frey and A.J. Baeumnerb, J. Membr. Sci., 279, 354 (2006).

16. F. Bibbs, ELISA Technical Bulletin-No. 3, Corning Life Sciences, Kennebunk, ME, USA, Retrieved web site, http://www.corning.com/ lifesciences

17. R.F. Vogt, D.L. Phillips Jr., L. Omar Henderson, W. Whitfield and F.W. Spierto, J. Immunol. Methods, 101, 43 (1987).

18. S.C. Lakhotia, A. Sharma, M. Mutsuddi and M.G. Tapadia, Trends Genet., 9, 261 (1993).

19. T. Lu, C. Deng, J. Sun, X. Chen, P. Zhang and X. Jing, Chem. J. Chin. Univ., 29, 837 (2008).

20. F. Rusmini, Z. Zhong and J. Feijen, Biomacromolecules, 8, 1775 (2007).

21. M. González, C. Argarana and G.D. Fidelio, Biomol. Eng., 16, 67 (1999). 